\title{
Perspectives d'enregistrement 3D d'une fouille archéologique sur plusieurs années : Le cas de la villa du "Grésil" (Seine-Maritime)
}

\section{Perspectives on 3D recording of an archaeological excavation through multiple years: The case of the villa of "Grésil" (Normandy, France)}

\author{
Rémi Méreuze ${ }^{1}$, Jérôme Spiesser ${ }^{2}$ \\ ${ }^{1}$ Université Paris 1 Panthéon-Sorbonne, UMR 8096 ArchAm, Archéologie des Amériques, MAE, 21, allée de l'Université, \\ F-92023, Nanterre, remi.mereuze@univ-paris1.fr \\ ${ }^{2}$ Université Paris 1 Panthéon-Sorbonne, UMR 7041 ArScAn, Equipe Archéologies environnementales, Archéologie des \\ Amériques, MAE, 21, allée de l'Université, F-92023, Nanterre, jerome.spiesser@hotmail.fr
}

RÉSUMÉ. Située au cœur d'une forêt, la villa gallo-romaine du « Grésil » (Seine-Maritime) est fouillée depuis 2012. Les conditions de conservations du site sont exceptionnelles puisque ce secteur a vraisemblablement été reboisé depuis l'abandon de l'habitat. Ce contexte particulier rend cependant très difficile l'acquisition de donnée photographique zénithale. Afin de remédier à cela, un protocole d'enregistrement 4D de l'évolution de la fouille a été mis en place dès 2013 et perfectionné au cours des années.

ABSTRACT. The Roman villa of "Gresil" (Seine-Maritime) is located inside a forest, the excavations began in 2012. The state of conservation of the site is exceptional since the place was apparently re-forested at its abandonment. It is, however, quite difficult to take zenith photos in this peculiar context. In order to work around the difficulty, a protocol of 4D records of the evolution of the excavation has been set since 2013 and perfected throughout the years.

MOTS-CLÉS. Photogrammétrie, Réalité virtuelle, Enregistrement 4D, Villa, Antiquité.

KEYWORDS. Photogrammetry, Virtual Reality, 4D recording, Villa, Antic period.

\section{Présentation du site}

Le site du «Grésil » est localisé à une quinzaine de kilomètres au sud de Rouen (Normandie), sur le plateau de l'un des méandres de la basse vallée de la Seine (figure 1). Les recherches archéologiques menées sur cet habitat gallo-romain ont commencé en 1903 à l'initiative de Léon de Vesly, puis ont repris en 2012. La particularité de cet établissement est d'être remarquablement bien conservé puisqu'il est situé au cœur d'un massif forestier ancien, probablement mis en place dès l'abandon du site. Bien que le sommet des maçonneries soit directement présent sous l'humus forestier, aucun stigmate lié au labour n'y a été décelé. Ce contexte boisé qui a permis la conservation des murs maçonnés sur $0,4 \mathrm{~m}$ d'élévation, possède toutefois l'inconvénient de rendre impossible la photographie aérienne du site par avion, cerf-volant ou drone. La réalisation de relevés photogrammétriques a initialement été mise en place afin de remédier à cela, et c'est progressivement perfectionné pour aboutir à un véritable protocole d'enregistrement $4 \mathrm{D}$ de l'évolution de la fouille. 


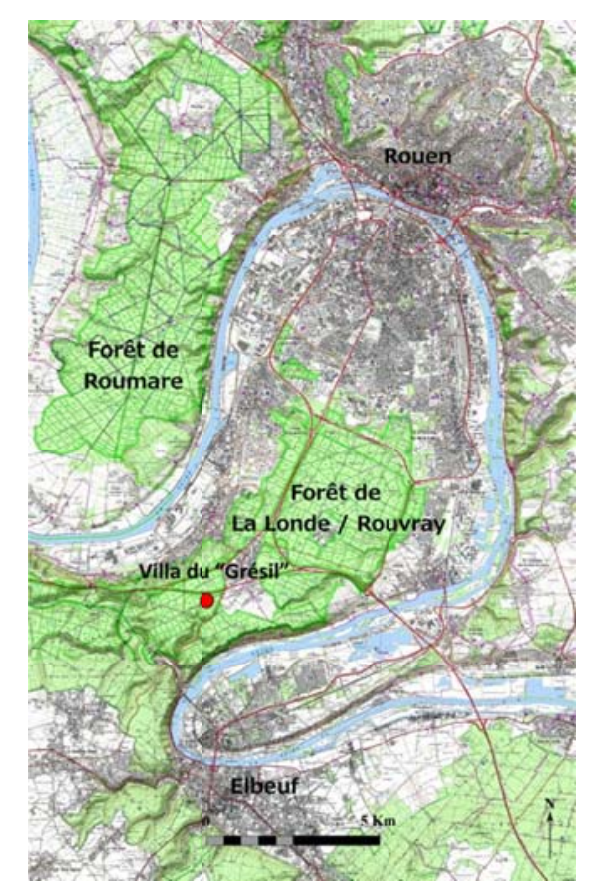

Figure. 1. Localisation de la villa du "Grésil" (D.A.O.: J. SPIESSER)

L'exploitation agricole du «Grésil» s'installe à la période augustéenne à $500 \mathrm{~m}$ de l'une des principales voies de communication de la cité des Véliocasses. Cet axe routier reliait la capitale Rotomagus (Rouen) à la petite agglomération secondaire d'Uggate (Caudebec-Lès-Elbeuf), actuellement localisée à la périphérie est de la ville d'Elbeuf. Cet établissement possède une pars urbana, composé d'un bâtiment résidentiel et de deux petites infrastructures liées au stockage (figure 2). Dans un premier temps, l'habitation est construite en terre et bois sur de petits solins en pierres sèches (figure 3). Elle mesure au moins $40 \mathrm{~m}^{2}$ divisés en deux pièces. Il faudra attendre la seconde moitié du $\mathrm{I}^{\mathrm{er}}$ siècle après J.-C. pour que ce bâtiment soit agrandi afin d'atteindre une superficie minimale de $75 \mathrm{~m}^{2}$. Ces nouveaux aménagements seront toujours réalisés en matériaux périssables, mais reposeront désormais sur de larges solins composés d'un amoncellement de petits silex. L'optimum du site se situe au milieu du $\mathrm{II}^{\mathrm{e}}$ siècle lors de la mise en place d'une enceinte maçonnée délimitant une pars urbana de $3000 \mathrm{~m}^{2}$, ainsi que l'installation de deux bâtiments de stockage aux angles nord et est de celle-ci. La pièce sud de la résidence est également réaménagée à cette occasion. Elle sera alors dotée d'un sol maçonné et d'un décor mural peint. Cette exploitation agricole dont la présence de faisselle laisse supposer la fabrication de produits laitiers sera abandonnée dans la première moitié du $\mathrm{III}^{\mathrm{e}}$ siècle.

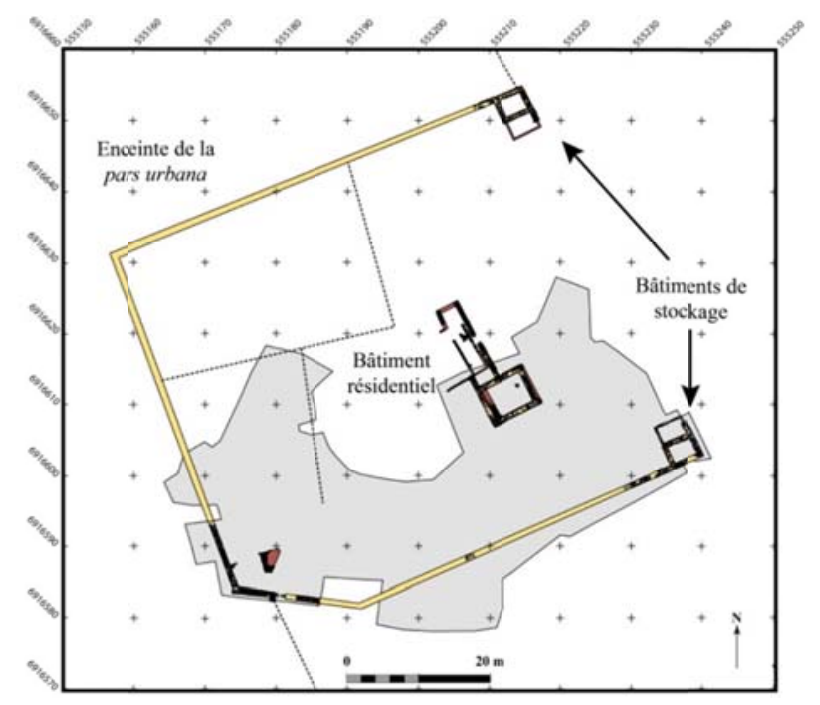

Figure 2. Plan du site du "Grésil" (D.A.O.: J. SPIESSER) 


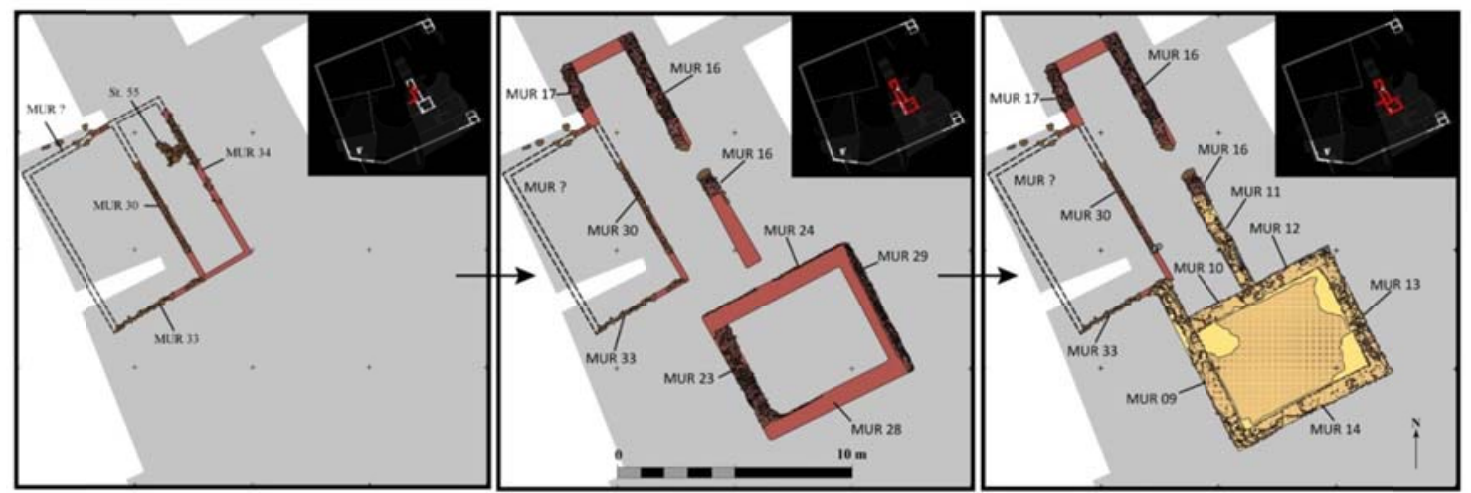

Figure. 3. Évolution architecturale du bâtiment résidentiel (D.A.O.: J. SPIESSER)

\section{Mise en place des relevés 3D}

Dans un premier temps, la modélisation 3D fut utilisée pour multiplier les techniques d'enregistrement et obtenir une vue zénithale des structures fouillées. L'utilisation de la 3D en archéologie possède de nombreux avantages comme l'étude des aires et des volumes (de Luca et Florenzano 2004). Il est aussi possible d'obtenir une vue orthographique des modèles enregistrés, c'est-à-dire de produire une image en plan sans effet de perspectives ${ }^{1}$ (Héno 2010). La possibilité de produire des vues pour en faciliter les relevés planimétriques fut un élément déterminant dans le choix d'utiliser la photogrammétrie sur le site du « Grésil». Cela permettait ainsi de relever rapidement les niveaux souvent considérés comme «secondaires » par les archéologues, par exemple ceux relatifs à la démolition du site. Ces modèles 3D complétaient également les premiers relevés topographiques effectués à l'aide d'une station totale. De plus en plus utilisée en archéologie, la photogrammétrie reste néanmoins une discipline récente. Son faible coût matériel et la rapidité d'exécution en sont les principaux atouts. Seuls un appareil photographique et un ordinateur (équipé des logiciels adéquats) sont a priori nécessaires pour obtenir des données fiables (Linder 2009). Dans le cas de la fouille du «Grésil », seules des prises en vues pédestres ont été réalisées afin de limiter le coût dédié aux équipements.

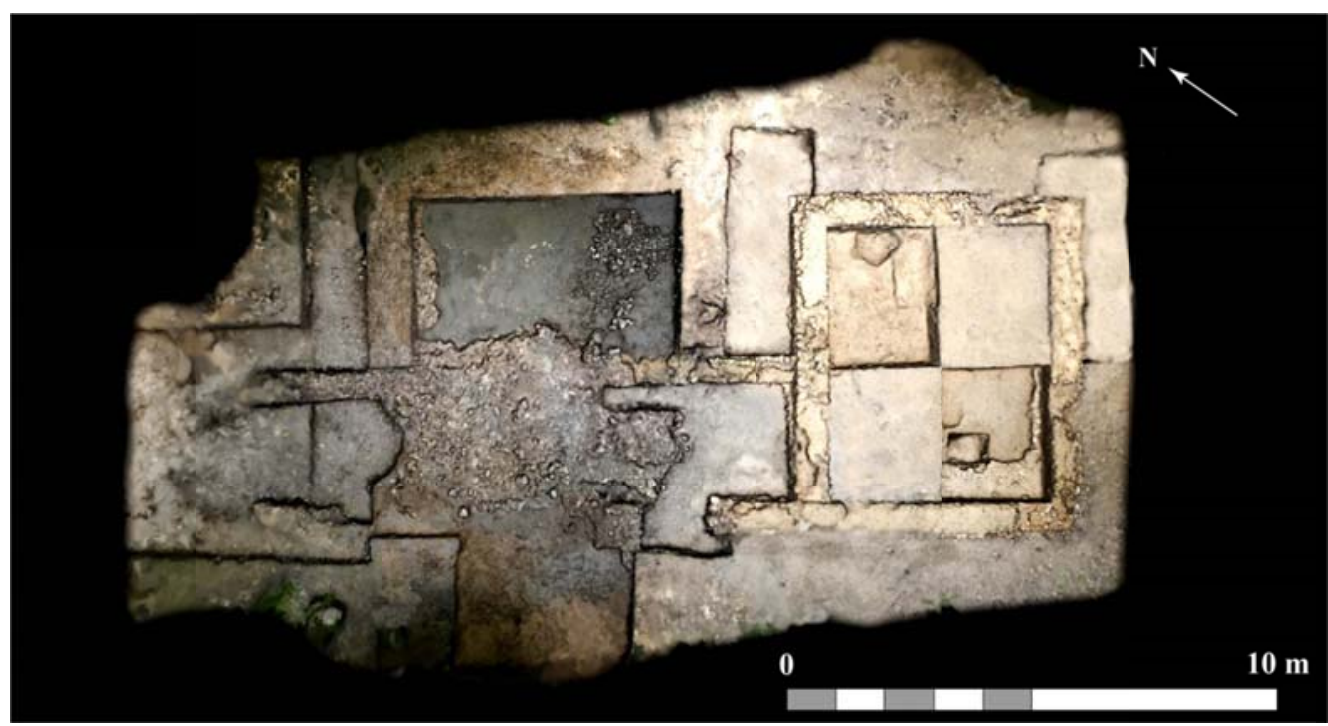

Figure. 4. Premier relevé photogrammétrique du site

(Photogrammétrie : R. MEREUZE, D.A.O.: J. SPIESSER)

\footnotetext{
${ }^{1}$ À ne pas confondre avec une photographie redressée qui correspond à une prise de vue (ayant subi un effet de perspective) déformée pour correspondre au plus prêt à une vue orthographique, sans en être réellement une (Kasser et Polidori 2001, p.282). (c) 2017 ISTE OpenScience - Published by ISTE Ltd. London, UK - openscience.fr 
L'année 2013 fut l'occasion de tester cette méthodologie en la confrontant aux relevés manuels afin de tester sa fiabilité. À cette occasion, le modèle 3D du bâtiment résidentiel (figure 4) a ainsi pu être comparé au dessin plus traditionnel (figure 5). Pour des raisons idéologiques et financières, le traitement photogrammétrique n'a été réalisé qu'avec des logiciels libres. Il n'est pas inutile de rappeler que le «libre» correspond à une volonté d'ouverture et d'accessibilité aux outils, aux méthodologies, mais également aux données. Les deux logiciels qui seront présentés ici sont par conséquent accessibles à toutes personnes souhaitant se former à la modélisation 3D. Dans un premier temps, l'assemblage des photos et le calcul du nuage de point ont été effectués grâce à Python Photogrammetry Toolbox (Moulon et Bezzi 2011) (basé sur osm-bundler). Le nettoyage du nuage, la mise à l'échelle et la création de la surface ont quant à eux été réalisés avec Meshlab, un logiciel développé par le $\mathrm{VCG}^{2}$ de l'ISTI ${ }^{3}$, une branche du $\mathrm{CNR}^{4}$ (Calieri et al. 2008).

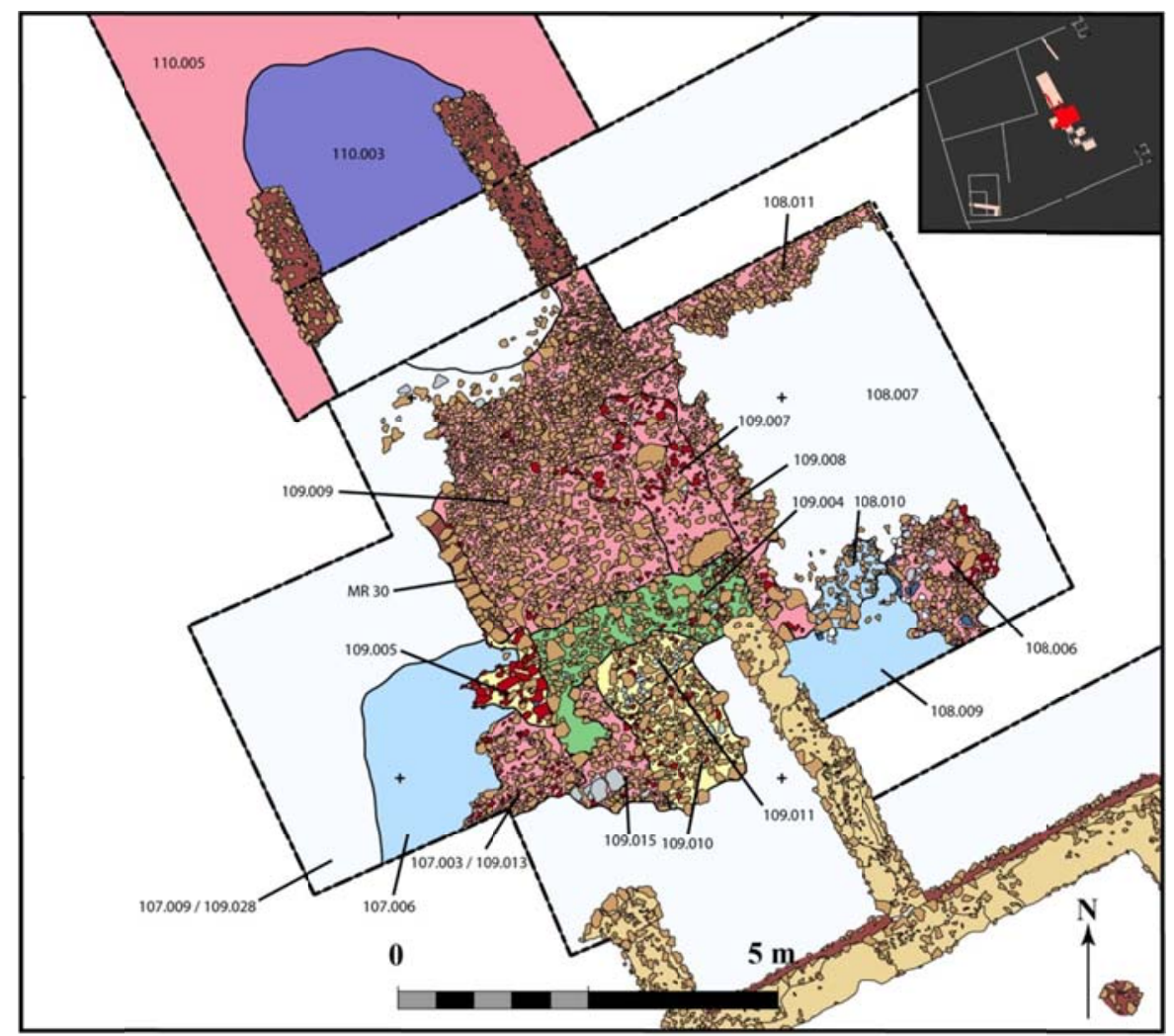

Figure 5. Relevé manuel de la zone modélisée en 3D par photogrammétrie (D.A.O.: J. SPIESSER)

L'une des difficultés majeures des relevés par photogrammétrie est la redondance des formes apparaissant sur les photographies. La répétition de motifs identiques sur un même cliché ou entre les différentes photographies peut empêcher le remontage des prises de vues entre elles (Kraus 2007). Cette difficulté s'est avérée particulièrement importante sous le couvert forestier à cause de l'omniprésence de feuilles, dont leurs morphologies analogues créent des redondances. Les premiers résultats furent par conséquent de qualités diverses, suite à la complexité du remontage des photographies entre elles. Pour pallier à cela, des plots orange et noir ont été disposés aléatoirement sur le terrain afin de briser les redondances et faciliter le recalage des clichés (figure 6).

Grâce à cette première modélisation, nous avons pu constater que les erreurs obtenues sur les modèles 3D étaient comparables aux relevés manuels. L'erreur la plus importante était de $2,5 \mathrm{~cm}$ pour une structure de $46 \mathrm{~m}^{2}$. La résolution des mesh ${ }^{5}$ était alors suffisante pour effectuer des relevés pierre à

\footnotetext{
${ }^{2}$ Visual Computing Lab (Laboratoire de Visualisation par Ordinateur).

${ }^{3}$ Instituto di Scienza e Tecnologie dell'Informazione (Institut des Sciences et Technologies de I'Information).

${ }^{4}$ Consiglio Nazionale delle Ricerche (Conseil National de la Recherche).

${ }^{5}$ Une surface 3D formée d'un maillage de polygones. 
pierres d'après orthophotographies, sans perdre d'information. Enfin, l'ajout des plots augmentait la robustesse de la méthode en limitant les risques d'échec de remontage des clichés photographiques.

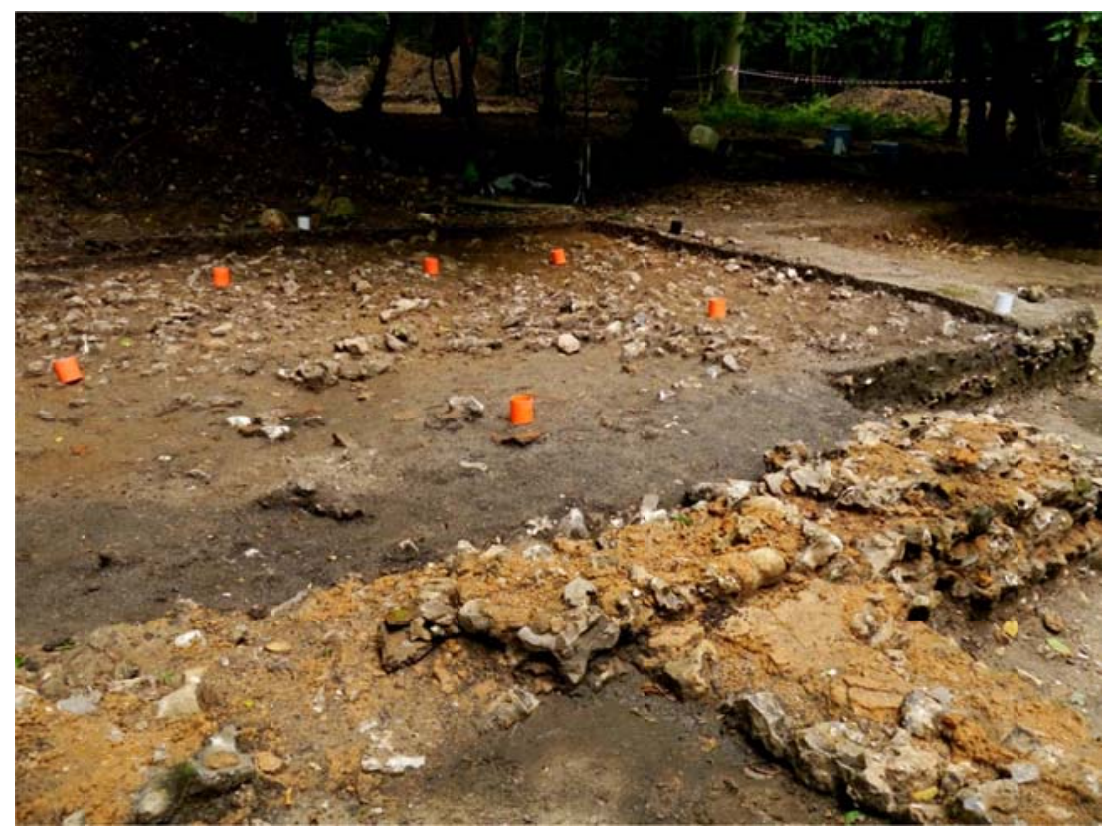

Figure 6. Photographie de l'installation de plots pour la photogrammétrie (Photographie : J. SPIESSER)

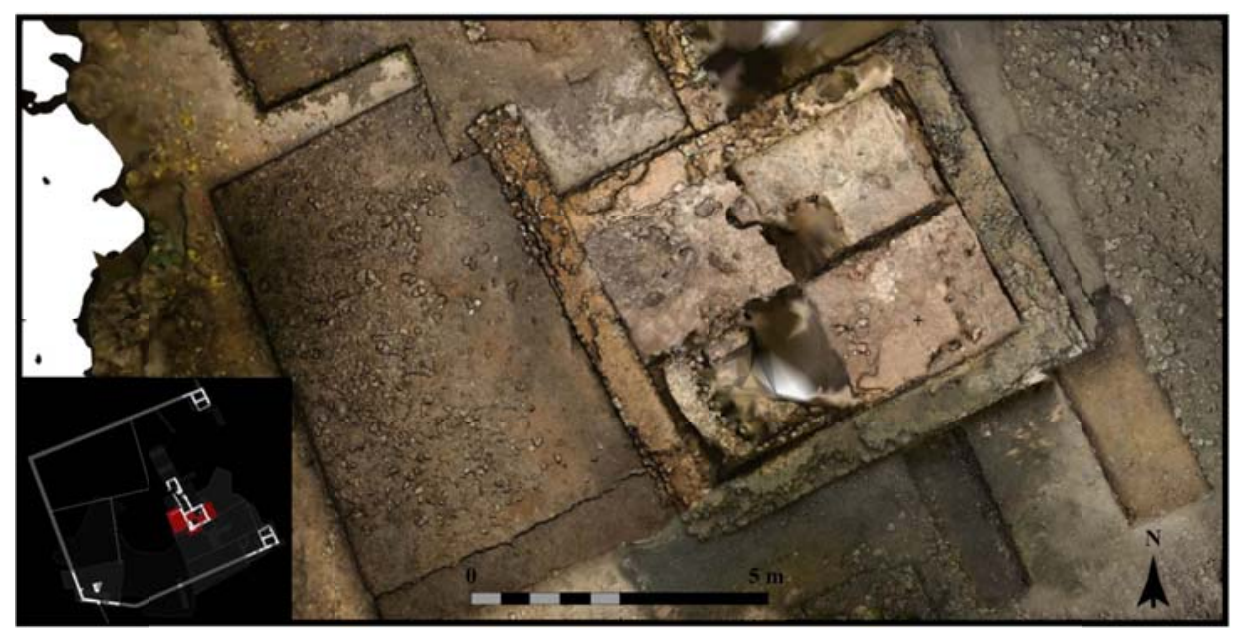

Figure 7. Relevé photogrammétrique avec le dispositif de plots (Photogrammétrie : R. MEREUZE, D.A.O.: J. SPIESSER)

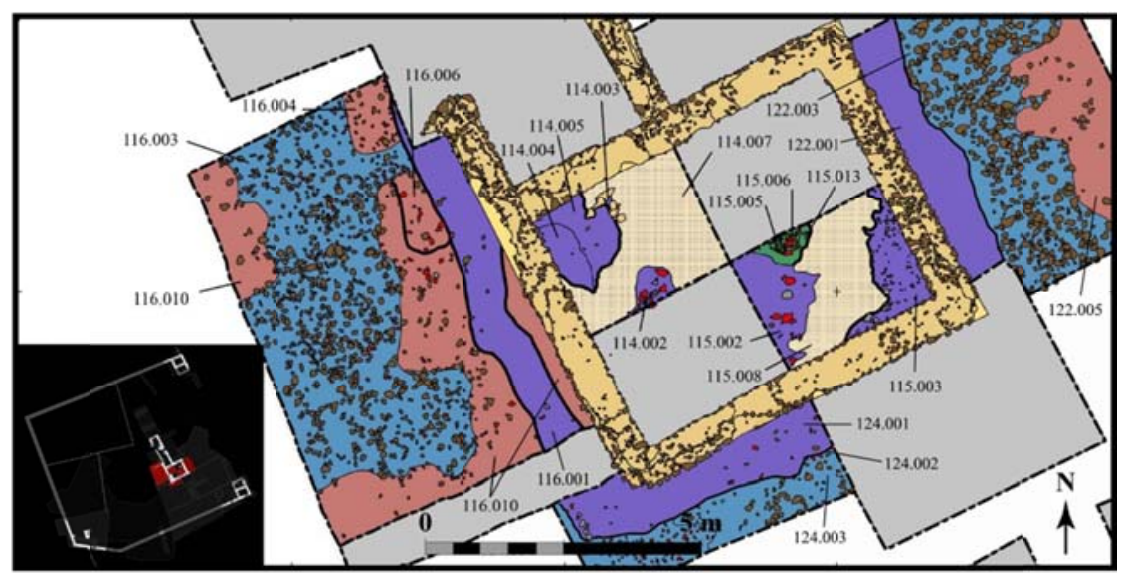

Figure 8. Relevé des niveaux de démolition à partir du modèle 3D (D.A.O.: J. SPIESSER) 


\section{Application à l'ensemble du site}

À partir de 2014, la photogrammétrie fut utilisée à grande échelle pour relever les structures fouillées, mais aussi à grande échelle afin d'obtenir une première vue zénithale du site. Cette nouvelle approche nécessite néanmoins la présence de sources de stockage annexe puisqu'à la fin du traitement, le volume de données peut doubler, voire tripler. Il est également indispensable d'alléger les nuages de points si l'on souhaite les compiler au sein d'un même espace virtuel. Nous avons donc intégré un logiciel non libre dans notre protocole. Photoscan fut choisi pour sa rapidité d'exécution et sa simplicité d'utilisation. Le nettoyage et surtout l'allègement des nuages de points ont été réalisés avec le logiciel CloudCompare (Girardeau-Montaut, D. 2011). Un premier modèle 3D de l'ensemble du site (figure 9 a) a par conséquent été réalisé en parallèle des différents relevés de structure. Ce modèle fut établi à partir de 296 clichés, couvrant une superficie de $1738 \mathrm{~m}^{2}$. L'ensemble des productions photogrammétriques a ensuite été associé dans un même espace virtuel (au sein du logiciel Meshlab) afin d'effectuer différentes analyses spatiales comme la réalisation d'un modèle numérique de terrain (figure 10).

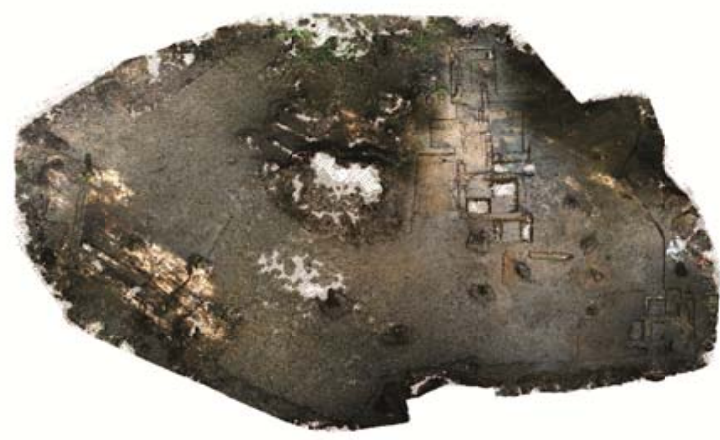

Vue "aérienne" en 2014

Figure 9 a. Premier modèle d'ensemble du site (Traitement : R. MEREUZE)

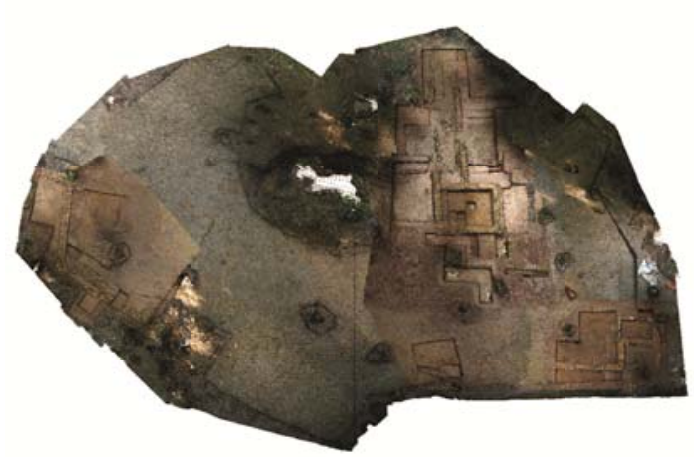

Vue réactualisée après lété 2015

Figure 9 b. En 2015, seules les parties fouillées ont été remplacées sur le précédent modèle

(Traitement : R. MEREUZE)

Dès le début de la campagne de 2015, un premier relevé des cinq secteurs de fouille a été opéré pour servir de base à l'ensemble des modèles qui seraient créés ultérieurement. Ces secteurs ont ensuite été superposés sur le précédent mesh général du site (figure $9 \mathrm{~b}$ ). Au total, 27 structures et étapes de fouille ont été enregistrées durant cette saison, et replacées dans un environnement 3D secteur par secteur (figure 11).

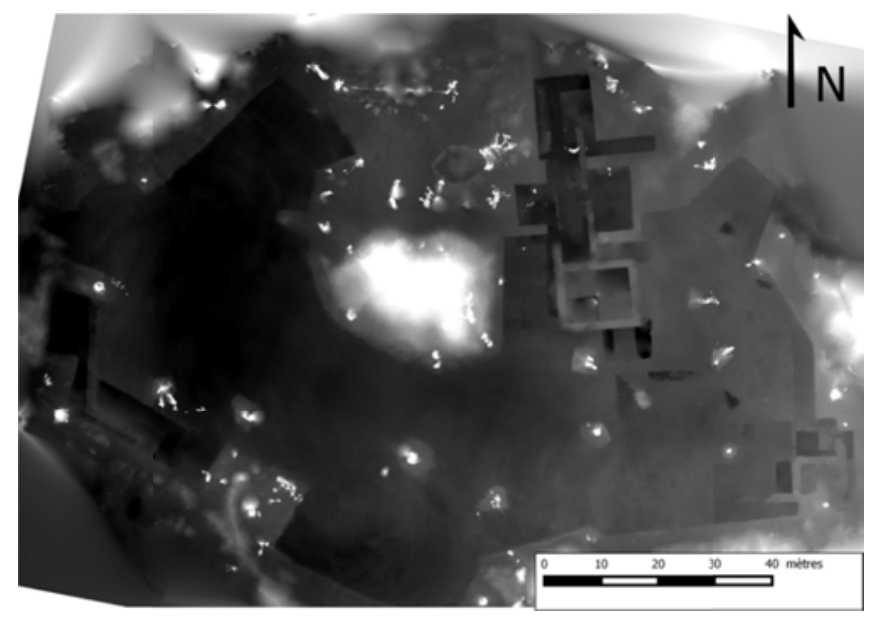

Figure 10. Modèle numérique de terrain réalisé à partir de la photogrammétrie (Traitement : R. MEREUZE) 


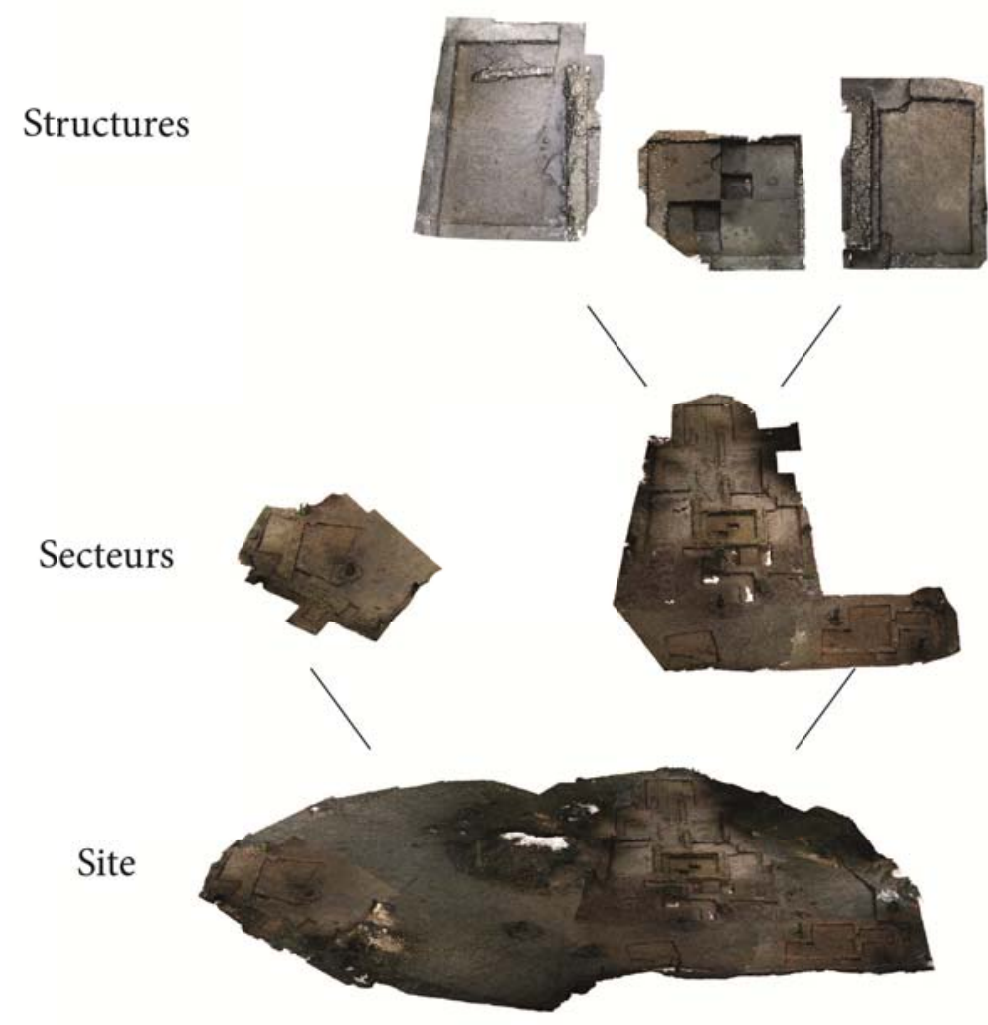

Figure 11. Emboitement des structures dans les secteurs et des secteurs dans le site (Traitement : R. MEREUZE)

\section{Vers un SIG 4D}

Un SIG 4D ${ }^{6}$ a progressivement été mis en place, c'est-à-dire un Système d'Information Géographique intégrant l'ensemble des surfaces 3D y compris celles qui se superposent. Celles-ci correspondent à des étapes de fouille différentes et, de fait, à des temps différents. La recherche d'un SIG 4D est actuellement très prégnante en archéologie, un tel outil permettant d'étudier une structure (Mathieu 2013), un site (Rollier et coll. 2014) ou une région (Scianna 2013), durant le temps de son occupation, afin d'en suivre l'évolution sous toutes les dimensions possibles. Parallèlement il permet de suivre l'évolution de la fouille elle-même, de replacer les faits archéologiques les uns par rapport aux autres (Samaan et coll. 2014) et de replacer les méthodes et techniques employées au cours de l'exploitation du site de fouille. Ces deux derniers aspects sont particulièrement intéressants à appliquer sur un site comme celui du «Grésil», sur lequel les stratégies de fouille et les méthodes employées étaient exploratoires et renouvelées chaque année. Dans l'objectif de caractériser au mieux l'occupation du site, une approche interdisciplinaire a été menée afin de comparer spatialement les différentes études, qu'elles soient archéologiques, pédologiques, géophysiques ou encore topographiques.

Pour l'instant cet outil est encore sous la forme d'un ensemble de mesh situé dans un système de coordonnées locales. Il sera bientôt relié au SIG général de la fouille. Le Modèle Numérique de Terrain reste cependant aisément productible. Grâce à l'enregistrement systématique en 3D des couches archéologiques, nous avons donc pu assurer un meilleur contrôle de la fouille et des différentes avancées du projet, puisqu'il devenait possible de déconstruire l'ensemble de la fouille au moyen de la réalité virtuelle (figure 12).

\footnotetext{
${ }^{6}$ Un SIG (Système d'Informations Géographiques) qui contient des données 3D et des informations temporelles, la quatrième dimension. 


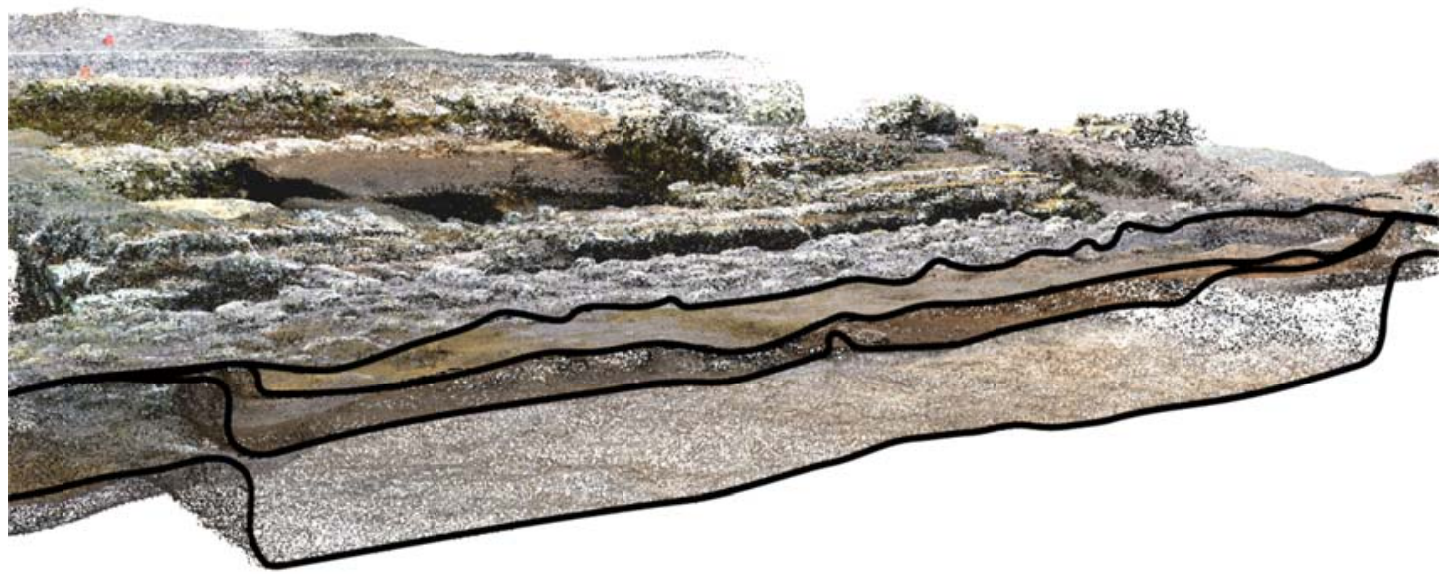

Figure 12. Section des couches superposées dans CloudCompare (Traitement : R. MEREUZE)

\section{Conclusions et perspectives}

À terme, le modèle 3D de la fouille du «Grésil» sera diffusé en accès libre pour en permettre la manipulation aux personnes intéressées. Ceci se fera à nouveau dans l'univers du «libre » à partir de 3DHOP (http://3dhop.net/). Développé par l'ISTI, il s'agit d'un ensemble de codes et d'outils pour la publication de modèles 3D en HTLM. Il autorise également l'annotation des modèles et la réalisation de sections. Par conséquent, il permet de revenir sur la succession stratigraphique d'une structure ou du site. Les visiteurs du site pourront alors visualiser cet ensemble de données sans avoir besoin d'installer ne serait-ce qu'une extension à leur navigateur web puisque tout est écrit en CSS/HTLM et en JAVA. Lié à un site internet dont la base de données correspond à celle du projet archéologique, 3DHOP nous permettra de publier le site avec la possibilité de manipuler l'ensemble des données (figure 13).

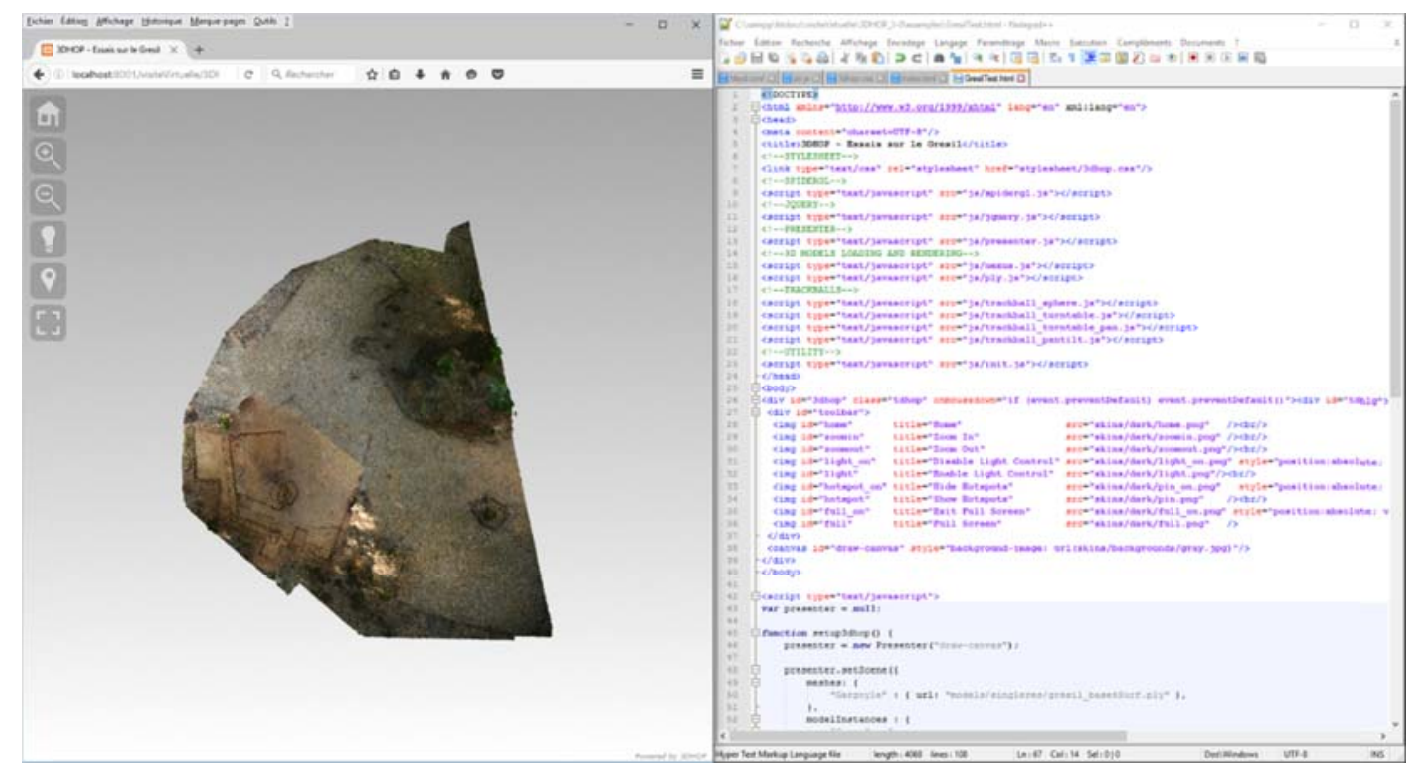

Figure 13. Les premiers tests dans le visualiseur HTML 3DHOP (Traitement : R. MEREUZE)

Parallèlement à la publication scientifique, la visualisation virtuelle du site est un excellent outil de médiation, d'autant que ce dernier ne fera l'objet d'aucun projet de valorisation. Les dernières structures existantes seront ré enfouis prochainement pour stabiliser leur dégradation. Concernant la médiation, il faut rappeler que la 3D possède l'avantage d'être un objet visuellement attractif et facile à comprendre. 
Les données 3D peuvent d'ores et déjà être employées pour permettre une visite virtuelle du site aux visiteurs intéressés. Les mesh utilisés pour l'analyse scientifique ne sont pas adaptés tels quels à une utilisation par le grand public. Ils sont en effet trop volumineux pour être affichés de façon fluide sur un ordinateur de puissance moyenne. De même, le rendu visuel peut déranger un public non averti, puisque les couleurs sont parfois absentes et le jeu de lumière surréaliste. Pourtant, ces données sont facilement modifiables avec des logiciels de modélisation 3D, tels que libre Blender (https://www.blender.org/). Il permettra de créer une cohérence visuelle pour l'ensemble de la 3D du site. Ce nouveau modèle sera ensuite calculé dans un moteur de rendu de jeux vidéo afin d'être visible de façon interactive. Nous utiliserons le logiciel Unity (https://unity3d.com/) qui reste gratuit sous un certain seuil d'utilisation. Il possède en outre un certain nombre d'extensions pour développer des applications adaptées aux différentes plateformes et outils de contrôle. Le modèle virtuel de la villa du «Grésil» est dès à présent adapté à l'usage de lunettes d'immersion. Là encore, il est possible de persévérer dans le «libre» grâce à l'OSVR (http://www.osvr.org/), dont les plans sont libres de droits et les logiciels de développement sous licence APACHE 2.0.

Avec cette nouvelle chaine d'outils, toujours dominée par l'emploi de logiciel libre, il est ainsi devenu possible d'adapter pour le grand public, les données acquises au cours des différentes années de fouille et de les rendre accessibles au moyen des nouvelles technologies de réalité virtuelle immersive.

\section{Conclusions}

Utilisée d'abord de façon exploratoire, la photogrammétrie s'est révélée être un outil primordial pour l'étude du site du Grésil. Ce site sous forêt a ainsi pu être observé dans son ensemble, depuis un ciel virtuel nous aidant à en mieux saisir l'emprise dans son environnement. Les surfaces de fouilles, comme les choix opérés aux cours de ces trois années de campagne, ont été remis en perspective les uns par rapport aux autres et il nous est devenu possible de revenir sur ces actions, même plusieurs années après. Grâce aux techniques de la 3D, chaque jour en évolution, des informations que nous pensions n'être applicables qu'à la documentation scientifique du projet ont finalement été adaptées pour des utilisations grands public. Cela accorde au site une existence après les fouilles, son modèle virtuel étant devenu visitable par chacun au moyen de navigateurs web ou même de lunette d'immersion. C'est donc sur plusieurs échelles spatiales, du site aux structures, et temporelles, de son enregistrement à sa visite, que la 3D est devenue un pilier du projet archéologique du Grésil.

\section{Bibliographie}

Cignoni, P., CAllieri, M., Corsini, M., Dellepiane, M., GANOvelli, F., \& RanZuglia, G., 2008. « Meshlab: an opensource mesh processing tool”, Eurographics Italian Chapter Conference, vol. 2008 : 129-136.

DE LuCA, L., \& M. FlorenZANO, 2004. «Relevé d'architecture-Du nuage de points à la maquette 3D, l'apport des modèles théoriques issus de l'histoire de la représentation.», MICAD, consulté en ligne le 03/09/16 à l'adresse https://hal.archives-ouvertes.fr/halshs-00264208/.

GiRARDEAU-MontaUt, D., 2011. Cloudcompare-open source project. OpenSource Project.

HENO, R. 2010. « Archéologie et photogrammétrie, les nouveaux potentiels », Géomètre, 2075 : 26 - 40.

Kasser, M. \& L. Polidori, 2003. «From the aerial image to orthography: Different levels of rectification”, Kasser M. \& Y. Egels (dir.), Digital photogrammetry : 282 - 288.

KRAUS, K., 2007. Photogrammetry: Geometry from Images and Laser Scans, $2^{\mathrm{e}}$ edition, Walter de Gruyter .

LINDER, W., 2009. Digital photogrammetry, Springer.

Mathieu, V., SANZ, S., \& BohBot, H. (2013, November). Acquisition des données archéologiques en 3D: vers un outil de gestion dynamique en 3D. In Virtual retrospect 2013 (pp. 107-112). Ausonius.

Moulon, P. \& L. BEZZI, 2011. «Python photogrammetry toolbox: a free solution for three-dimensional documentation", ArcheoFoss : 1-12. 
Rollier, J., Z. Petty, A. Mazuir, S. Faucher, J. F. Coulais, \& G. Rollier, 2014. «Développement d'un SIG 4D pour la ville médiévale de Cluny », Archeologia e Calcolatori, Vol. Suppl. 5 : 164-179.

Samaan, M., M. Pierrot-Deseilligny, R. Heno, C. Montoya, \& S. Rassat 2014. « La Photogrammétrie rapprochée pour la modélisation en 4D d'une structure archéologique », Revue Française de Photogrammétrie et de Télédétection, $207: 59-70$.

SCIANNA, A., 2013. « Building 3D GIS data models using open source software », Applied Geomatics, 5 (2): 119-132. 